\title{
Photonic Gas Sensor Using a Silicon Strip Waveguide ${ }^{\dagger}$
}

\author{
Christian Ranacher 1,* , Andreas Tortschanoff 1, Cristina Consani 1, Mohssen Moridi 1, \\ Thomas Grille ${ }^{2}$ and Bernhard Jakoby ${ }^{3}$ \\ 1 Carinthian Tech Research AG, 9524 Villach, Austria; andreas.tortschanoff@ctr.at (A.T.); \\ cristina.consani@ctr.at (C.C.); mohssen.moridi@ctr.at (M.M.) \\ 2 Infineon Technologies Austria AG, 9500 Villach, Austria; thomas.grille@infineon.com \\ 3 Johannes Kepler University, Institute for Microelectronics and Microsensors, 4040 Linz, Austria; \\ bernhard.jakoby@jku.at \\ * Correspondence: christian.ranacher@ctr.at; Tel.: +43-4242-56300-237 \\ + Presented at the Eurosensors 2017 Conference, Paris, France, 3-6 September 2017.
}

Published: 25 August 2017

\begin{abstract}
Sensing of gases is a promising area for applications of photonic systems operating in the mid-infrared spectral range. We present an infrared evanescent-field absorption gas sensor based on a silicon strip waveguide, which was specifically designed for $\mathrm{CO}_{2}$ sensing. We discuss finite element simulations that were used to design the strip waveguide and furthermore present experimental data of quantitative $\mathrm{CO}_{2}$ measurements with the devised structures. The first demonstrator device detects concentrations down to $5000 \mathrm{ppm} \mathrm{CO}_{2}$ which is the workplace exposure limit in most jurisdictions.
\end{abstract}

Keywords: infrared gas sensing; silicon waveguide; evanescent-field absorption

\section{Introduction}

We present an infrared-absorption sensor for gas detection based on evanescent-field absorption using a silicon strip waveguide. Due to the sub-wavelength dimensions of the dielectric waveguide, a small fraction of the electromagnetic (EM) modes that propagate along the waveguide also extend outside of the waveguide. The amount of this so called evanescent field, expressed in terms of the evanescent field ratio (EFR), depends on the cross section of the waveguide with respect to the wavelength of the propagating light, as well as on the optical properties of the used materials [1]. A high EFR and low intrinsic damping is crucial for gas sensing since the absorption coefficients are rather low for most gases. If an absorbing medium is brought in the vicinity of the waveguide, the evanescent field is absorbed by the gas attenuating the guided wave, which is detected in terms of a reduced intensity at the output port of the waveguide. Using waveguides for sensors allows incorporating long interactions paths for gas sensing into a small area by using structures like spirals, meanders, or resonators. Small dimensions are crucial for semiconductor mass production and our goal is to develop a fully integrated CMOS compatible sensor.

\section{Modeling and Design}

The devised sensor test structures are silicon strip waveguides with a length of about $1 \mathrm{~cm}$ which were designed for $\mathrm{CO}_{2}$ sensing. A taper and a launch-pad were added on both ends of the waveguide. On the launch-pad, a grating coupler is placed in order to couple mid-infrared radiation (MIR) from an external MIR source into the waveguide and to out-couple the light onto a detector. The design of the grating coupler, using two dimensional finite element models, was described in [2]. Figure 1 shows a schematic representation of the measurement configuration and a gas cell with a test chip, as it was used for the experiments. 

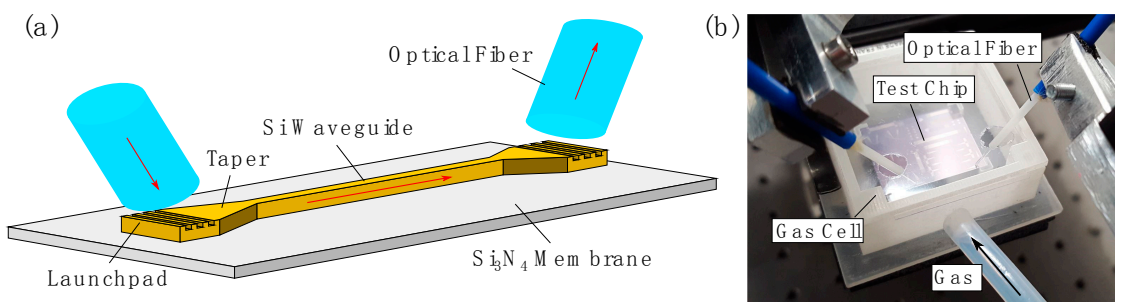

Figure 1. (a) Schematic representation of the measurement configuration; (b) Gas cell with a test chip and optical fibers. The gas enters the cell at the gas inlet. The holes for access of the optical fibers are used as an outlet for the gas.

The sensor structures were designed for a wavelength of $\lambda=4.26 \mu \mathrm{m}$ which is at the center of the $\mathrm{CO}_{2}$ absorption band. It is expected that an integrated IR source which could be used in the final sensor system will have a rather wide bandwidth that covers the whole $\mathrm{CO}_{2}$ absorption band. Therefore one needs to design the sensor for the center wavelength of the $\mathrm{CO}_{2}$ absorption band. A model of the strip waveguide was developed using the finite element method simulation tool COMSOL Multiphysics 5.2a. The material parameters were obtained from the literature [3,4]. A mode analysis was carried out on the cross-section of the waveguide. Figure 2 shows the normalized electric field distribution of the dominant coordinate $|E x|$ and $|E y|$, respectively, for the fundamental quasi-TE (Figure 2a) and the fundamental quasi-TM mode (Figure $2 b$ ). The silicon strip waveguide is located on a thin $\mathrm{Si}_{3} \mathrm{~N}_{4}$ membrane and the rest of the domain is filled by air/gas. For the simulation the domain size was chosen much bigger than shown in Figure 2 to avoid spurious effects at the boundary of the simulation domain.

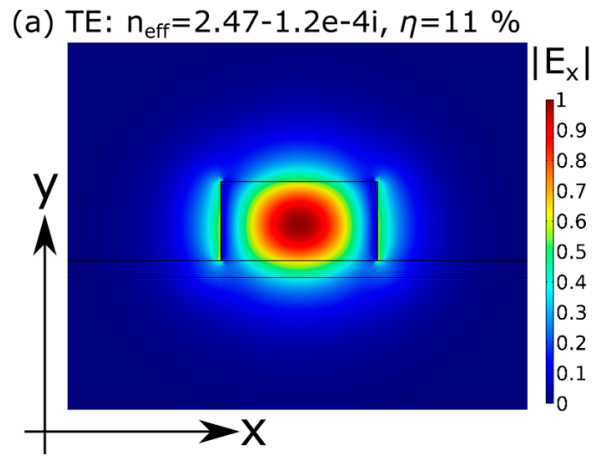

(b) TM: $\mathrm{n}_{\text {eff }}=1.75-4.3 \mathrm{e}-4 \mathrm{i}, \eta=21 \%$

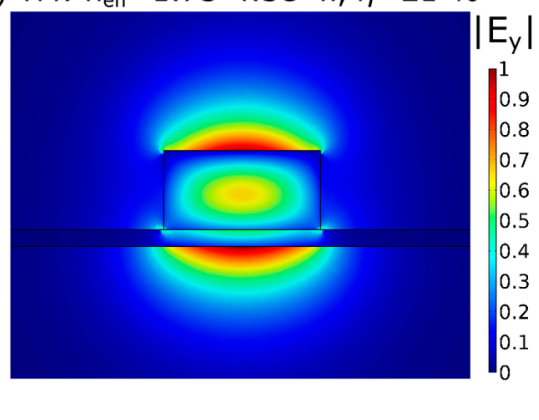

Figure 2. (a) Field distribution of $|\mathrm{Ex}|$ for the fundamental quasi-TE mode; (b) Field distribution of $\mid$ Ey| for the fundamental quasi-TM mode.

The (bulk) refractive index of a material $n=n^{\prime}-i \cdot n^{\prime \prime}$ is a complex material property. It consists of the real part $n^{\prime}$ which indicates the phase velocity or the wavenumber of a plane wave, in said material, and an imaginary part characterized by $n^{\prime \prime}$, the so called extinction coefficient, which accounts for attenuation (absorption) in the material. The wavenumber and the attenuation of a guided mode can in a similar manner be related to an effective index $\mathrm{n}_{\text {eff }}$ and the condition for guided modes is given by [1]:

$$
n_{\text {waveguide }}^{\prime}>n_{\text {eff }}^{\prime}>n_{\text {cladding }}^{\prime}
$$

If $n^{\prime}$ eff is smaller than $n_{\text {cladding, }}^{\prime}$ the mode becomes unguided meaning the EM field is oscillatory and not exponentially decaying (evanescent) in the cladding region, leading to an EM field that leaks out into a radiation mode. From the simulation the effective index was evaluated for the two modes and is also shown in Figure 2. At $\lambda=4.26 \mu \mathrm{m}$ the real part of the refractive index for $\mathrm{Si}_{3} \mathrm{~N}_{4}$ is $n^{\prime}=2.38$ [3]. According to Equation (1), the EM field of the fundamental quasi-TE mode $\left(n^{\prime}=2.47\right)$ is therefore oscillatory in the silicon strip waveguide only and it is evanescent in the $\mathrm{Si}_{3} \mathrm{~N}_{4}\left(n^{\prime}=2.38\right)$ and gas/air region $\left(n^{\prime}=1\right)$. The situation for the fundamental quasi-TM is different since $n^{\prime}=1.75<2.38$. 
Thus the quasi-TM mode also extends to the lossy $\mathrm{Si}_{3} \mathrm{~N}_{4}$ membrane. As a consequence the quasi-TM mode has an extinction coefficient that is almost four times higher than the extinction coefficient of the quasi-TE mode. Therefore the transmitted power over the $1 \mathrm{~cm}$ long waveguide of the quasi-TM mode is negligible. The EFR $\eta$ is a measure for the fraction of the EM field that can be used for absorption sensing. It was evaluated from the simulation results as the ratio of the field power in the air/gas area to the total modal power [5]. The evaluated $\eta$ for the two modes is also shown in Figure 2. The quasi-TM mode reaches almost twice the $\eta$ as the quasi-TE, but as already explained, the transmitted power of the quasi-TM mode is negligible and it will not contribute to sensing.

\section{Experimental}

The structures were fabricated on eight inch Si wafers, using standard semiconductor processes leading to polycrystalline silicon strip waveguides on a $\mathrm{Si}_{3} \mathrm{~N}_{4}$ membrane. The membrane was located on a $\mathrm{Si} / \mathrm{SiO}_{2}$ support structure. The fabrication of structures with a similar layer configuration is described in detail in [6].

The $\mathrm{CO}_{2}$ measurements were carried out using a custom test-bench which was described in [7]. For the quantitative gas measurements, the whole test chip was placed in a 3D printed gas cell (acrylate-type plastic) which was flushed with $\mathrm{CO}_{2} / \mathrm{N}_{2}$ mixtures at a total flow rate of $100 \mathrm{~mL} / \mathrm{min}$ (see also Figure 1). To account for the free-beam absorption between the fiber and the launch-pad which happens on both ends of the sensor, a reference measurement was performed and the results were corrected accordingly.

\section{Results and Discussion}

For the quantitative measurements, the QCL was tuned to $2362.8 \mathrm{~cm}^{-1}$ which corresponds to a wavelength of $\lambda=4.23 \mu \mathrm{m}$ and is the position of the $\mathrm{CO}_{2}$ line with the highest absorption. The time dependent intensity at the output port was recorded, while the gas cell was successively flushed with pure $\mathrm{N}_{2}$ and a $\mathrm{N}_{2} / \mathrm{CO}_{2}$ mixture. Although the system reacts immediately to a change of gas concentration due to the sensing principle, every mixture was flushed for a rather long time of $10 \mathrm{~min}$ through the cell since a low total flow rate of $100 \mathrm{~mL} / \mathrm{min}$ was used. The result is plotted in Figure 3a.
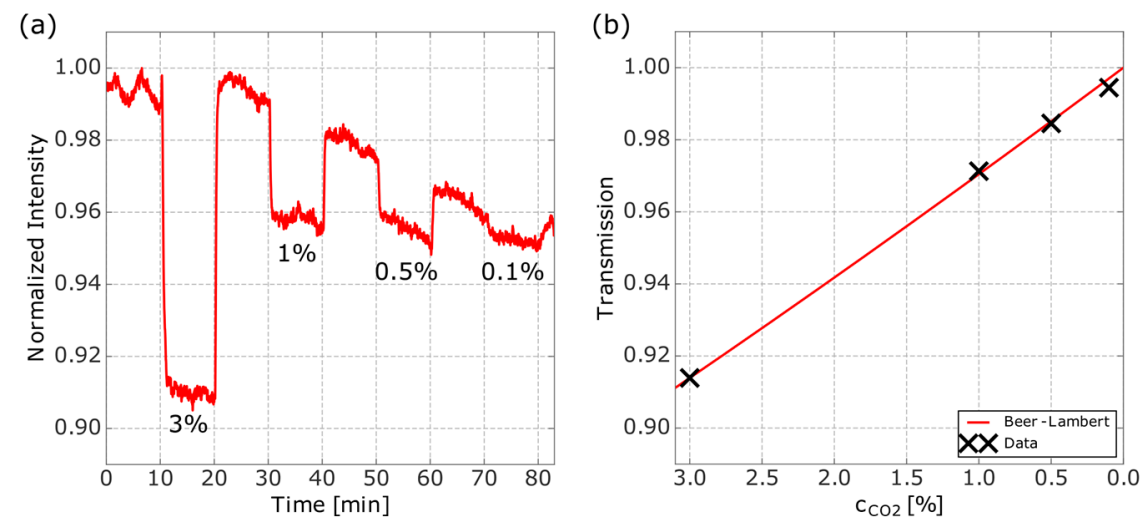

Figure 3. (a) Quantitative $\mathrm{CO}_{2}$ measurements. The labels below the data line give the concentration of $\mathrm{CO}_{2}$ in the gas mixture; (b) Measured transmission compared to the predicted transmission calculated using the Beer-Lambert law.

The baseline intensity decreased during the measurement due to micro-drift of the used stages (which was not eliminated in Figure 3a). The results show that a concentration of $0.5 \%$ (or $5000 \mathrm{ppm}$ ) $\mathrm{CO}_{2}$ can be detected. This is a significant improvement of one order of magnitude, compared to previous work where silicon slab waveguides were used [7]. Even for $0.1 \%$ the change in intensity is visible but close to the noise level. The transmission was calculated for the different $\mathrm{CO}_{2}$ concentrations, where the baseline drift was taken into account. The result is plotted in Figure 3b. 
The Beer-Lambert law adapted for our sensor was used to evaluate the possibility for quantitative measurements and converted in order to calculate the absorption coefficient $\alpha$ :

$$
I=I_{0} \cdot e^{-\eta \cdot c \cdot \alpha \cdot z} \rightarrow \alpha=-\frac{\ln \left(\frac{I}{I_{0}}\right)}{\eta \cdot c \cdot z}=-\frac{\ln (T)}{\eta \cdot c \cdot z}
$$

The parameters that were used to calculate $\alpha$ are the transmission $T=0.91$, which is the ratio of the transmitted power and the initial power, which was measured for a $\mathrm{CO}_{2}$ concentration of $c=3 \%$, the evanescent field ratio $\eta=11 \%$ (for the quasi-TE mode) and the path length $z=1 \mathrm{~cm}$ (which is the length of the strip). The absorption coefficient was determined as $\alpha=27 \mathrm{~cm}^{-1}$ and with this the transmission according to the Beer-Lambert law was calculated. This is in good agreement with the other experimental data-points as shown in Figure 3b.

When estimating an effective absorption coefficient from literature data [8] taking into account the exact center-wavelength and linewidth of the laser spectrum we obtain an estimated value of $\alpha_{\text {est }}=74 \mathrm{~cm}^{-1}$. The absorption coefficient evaluated from the experimental data was two to three times less than the estimated one, which is probably caused by an EFR which is lower than shown by simulation. This can be due to several reasons, including slightly different dimensions of the fabricated structures and material parameters that differ from literature values.

\section{Conclusions}

In this work we presented a gas sensor using a sub-wavelength silicon strip waveguide on a $\mathrm{Si}_{3} \mathrm{~N}_{4}$ membrane. The strip waveguides were designed using a finite element method model. The sensor was fabricated with standard silicon MEMS technology and is therefore suitable for semiconductor mass production. Quantitative $\mathrm{CO}_{2}$ measurements were performed down to $5000 \mathrm{ppm}$, which is the workplace exposure limit in most jurisdictions. Future work will investigate the intrinsic damping properties of such waveguides in order to be able to enhance the interaction length and therefore to further improve the performance of the sensor.

Acknowledgments: This project has been supported by the COMET K1 centre ASSIC Austrian Smart Systems Integration Research Center and the K2 centre LCM. The COMET-Competence Centers for Excellent Technologies-Programme is supported by BMVIT, BMWFW and the federal provinces of Carinthia, Styria, and Upper Austria. The authors would like to thank Infineon Technologies Austria AG, especially Surabhi Lodha and Markus Bergmeister for fabrication of the structures, as well as Thomas Ostermann for the support with the mask layouts.

Conflicts of Interest: The authors declare no conflict of interest. The funding sponsors had no role in the design of the study, in the collection, analyses, or interpretation of data, in the writing of the manuscript, and in the decision to publish the results.

\section{References}

1. Ghatak, A.; Thyagarajan, K. An Introduction to Fiber Optics; Cambridge University Press: Cambridge, UK, 1998.

2. Ranacher, C.; Consani, C.; Maier, F.J.; Hedenig, U.; Jannesari, R.; Lavchiev, V.; Grille, T.; Jakoby, B. Spectroscopic Gas Sensing Using a Silicon Slab Waveguide. Procedia Eng. 2016, 168, 1265-1269.

3. Kischkat, J.; Peters, S.; Gruska, B.; Semtsiv, M.; Chashnikova, M.; Klinkmüller, M.; Flores, Y. Mid-infrared optical properties of thin films of aluminum oxide, titanium dioxide, silicon dioxide, aluminum nitride, and silicon nitride. Appl. Opt. 2012, 51, 6789-6798.

4. Edwards, D.F.; Ochoa, E. Infrared refractive index of silicon. Appl. Opt. 1980, 19, 4130-4131.

5. Huang, Y.; Kalyoncu, S.K.; Zhao, Q.; Torun, R.; Boyraz, O. Silicon-on sapphire waveguides design for midIR evanescent field absorption gas sensors. Opt. Commun. 2014, 313,186-194.

6. Hedenig, U.; Grille, T.; Ritchie, G.A.D.; Kirkbride, J.M.; Jakoby, B.; Lavchiev, V. Characterization of Si Mid-Infrared Photonic Components for Chemical and Gas Sensing. In Proceedings of the AMA Sensors 2015, Dresden, Germany, 19-21 May 2015. 
7. Ranacher, C.; Consani, C.; Hedenig, U.; Grille, T.; Lavchiev, V.; Jakoby, B. A photonic silicon waveguide gas sensor using evanescent-wave absorption. In Proceedings of the 2016 IEEE SENSORS, Orlando, FL, USA, 30 October-3 November 2016; pp. 1-3.

8. Hitran. Available online: http://hitran.org/ (accessed on 6 July 2016).

(c) (1) BY
(C) 2017 by the authors. Licensee MDPI, Basel, Switzerland. This article is an open access article distributed under the terms and conditions of the Creative Commons Attribution (CC BY) license (http://creativecommons.org/licenses/by/4.0/). 